\title{
Kinetics and Mechanism of the Antioxidant Activities of $C$. olitorius and $V$. amygdalina by Spectrophotometric and DFT methods
}

Olaniyi K. Yusuff ${ }^{1}$, Modinah Adenike O. Abdul Raheem ${ }^{1 *}$, Abdulrahman A. Mukadam ${ }^{1}$, Ridwan Oladayo Sulaimon ${ }^{2}$

${ }^{1}$ Department of Chemistry, Faculty of Physical Sciences, University of Ilorin, PMB 1515, IlorinNigeria

${ }^{2}$ Department of Chemistry, King Fahd University of Petroleum and Minerals, P.O. Box 5061, Dhahran, Saudi Arabia

*Corresponding Author- Email: amadenike@unilorin.edu.ng: modinah4@yahoo.co.uk

Phone number: +2348035952356 


\section{Supporting Information}

Table s1: \% Inhibition of $V$. amygdalina at different concentrations

\begin{tabular}{|c|c|c|c|c|}
\hline $\begin{array}{l}\text { Concentration } \\
(\mathrm{mg} / \mathrm{ml})\end{array}$ & $\begin{array}{l}\text { \% Inhibition } \\
\text { (30 mins) }\end{array}$ & $\begin{array}{l}\text { \% Inhibition } \\
\text { (50 mins) }\end{array}$ & $\begin{array}{l}\text { \%Inhibition } \\
\text { (70 mins) }\end{array}$ & $\begin{array}{l}\text { \%Inhibition } \\
\text { (90 mins) }\end{array}$ \\
\hline 0.5 & 97.67 & 97.83 & 98.29 & 98.60 \\
\hline 0.25 & 96.20 & 96.66 & 96.97 & 97.44 \\
\hline 0.125 & 95.26 & 95.57 & 96.04 & 96.35 \\
\hline 0.0625 & 93.63 & 93.94 & 94.41 & 94.72 \\
\hline 0.0313 & 91.15 & 91.61 & 91.92 & 92.39 \\
\hline 0.0156 & 89.21 & 89.44 & 89.75 & 90.06 \\
\hline 0.00781 & 87.03 & 87.34 & 87.66 & 87.97 \\
\hline
\end{tabular}

Table s2: \% Inhibition of C.olitorius at different concentrations

\begin{tabular}{|c|c|c|c|c|}
\hline $\begin{array}{l}\text { Concentration } \\
(\mathrm{mg} / \mathrm{ml})\end{array}$ & $\begin{array}{l}\text { \% Inhibition } \\
(30 \text { mins })\end{array}$ & $\begin{array}{l}\text { \% Inhibition } \\
(50 \text { mins })\end{array}$ & $\begin{array}{l}\text { \%Inhibition } \\
(70 \text { mins })\end{array}$ & $\begin{array}{l}\text { \%Inhibition } \\
(90 \text { mins })\end{array}$ \\
\hline 0.5 & 92.69 & 93.32 & 94.28 & 94.91 \\
\hline 0.25 & 91.42 & 92.05 & 93.00 & 93.64 \\
\hline 0.125 & 89.83 & 90.78 & 91.42 & 92.37 \\
\hline 0.0625 & 87.28 & 87.92 & 88.87 & 89.51 \\
\hline 0.0313 & 81.72 & 82.35 & 83.31 & 83.94 \\
\hline 0.0156 & 76.15 & 77.11 & 77.74 & 78.38 \\
\hline 0.00781 & 68.36 & 69.32 & 69.95 & 70.91 \\
\hline & & & & \\
\hline
\end{tabular}




\section{DPPH Calibration Curve}

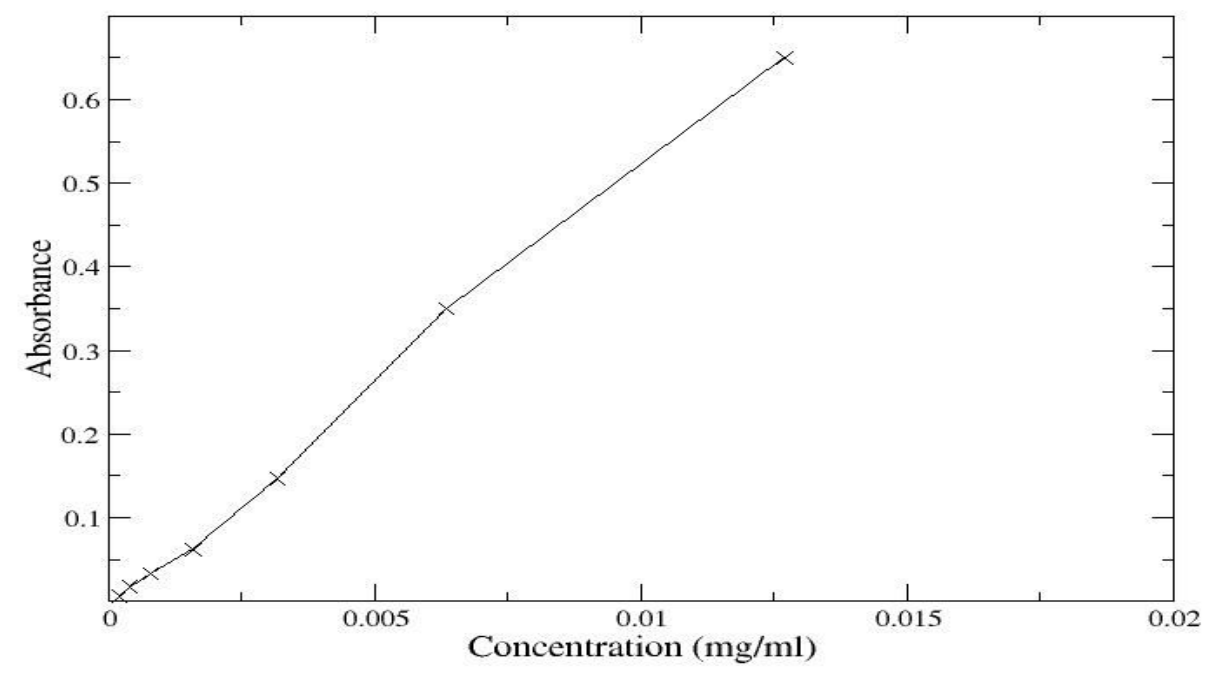

Figure s1: DPPH Calibration curve 


\section{(a) V. amygdalina pseudo first order rate constant}

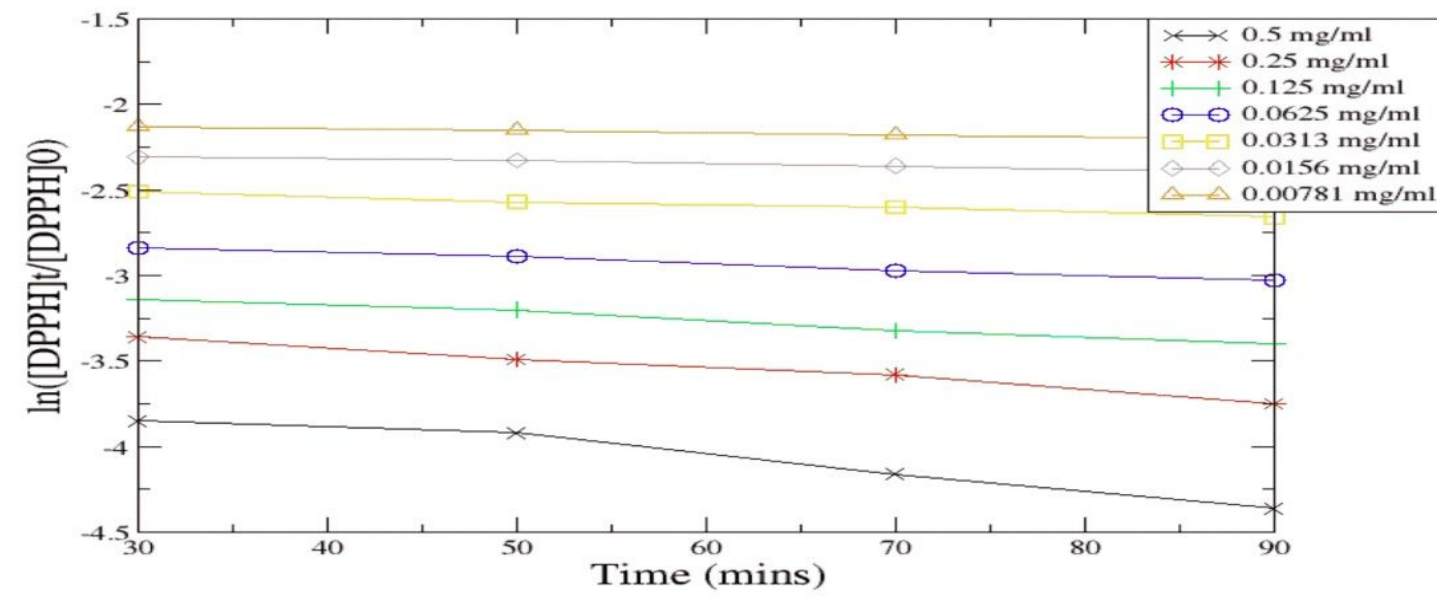

(b) C. olitorius pseudo first order rate constant

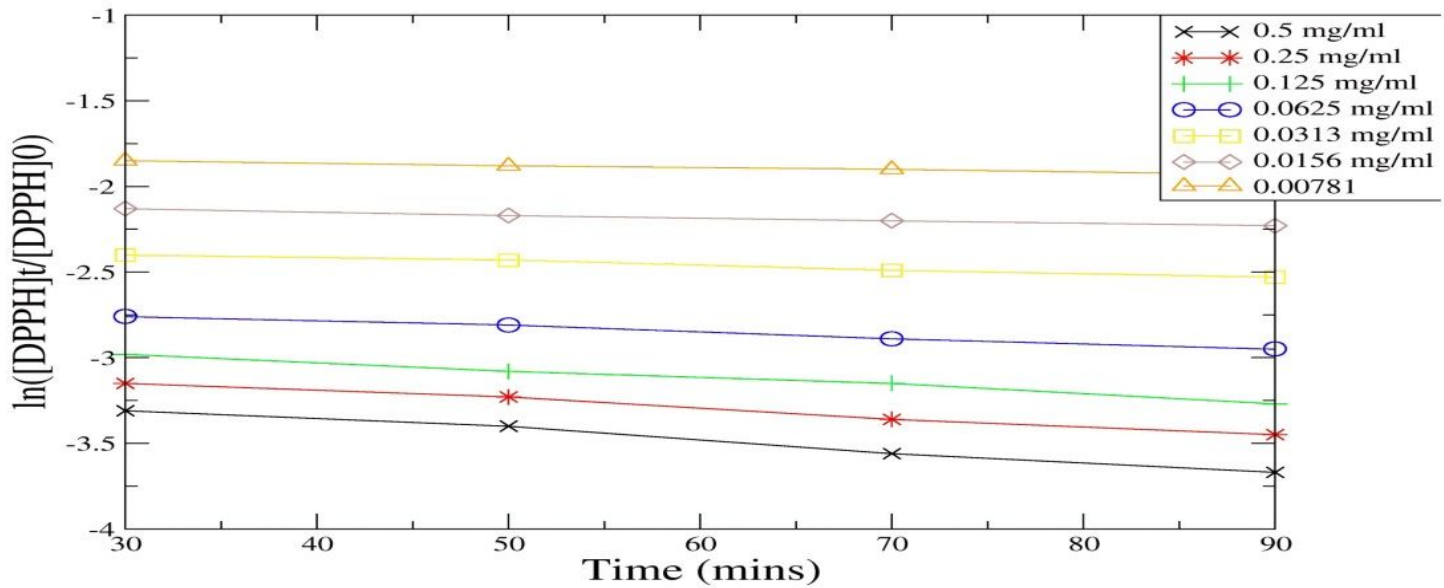

Figure s2:V. amygdalina (a) and C. olitorius (b) pseudo-first order rate constant 
Scheme s1: Cartessian coordinates of the optimized parent compound of Chlorogenic acid.

43

$\begin{array}{lrrr}\mathrm{C} & -1.44061000 & 0.68898400 & 1.87938900 \\ \mathrm{C} & -1.59237800 & 1.85568000 & 0.88100200 \\ \mathrm{C} & -2.90277000 & 1.74652800 & 0.08867400 \\ \mathrm{C} & -2.99358000 & 0.38678900 & -0.64708300 \\ \mathrm{C} & -2.89857400 & -0.77433200 & 0.35792900 \\ \mathrm{C} & -1.66490500 & -0.71007300 & 1.27009500 \\ \mathrm{H} & -3.75164000 & 1.84795900 & 0.77125500 \\ \mathrm{H} & -3.77868900 & -0.73938200 & 1.00655600 \\ \mathrm{O} & -0.46392700 & 1.96738200 & 0.01863200 \\ \mathrm{H} & -0.64246000 & 1.38070000 & -0.74348600 \\ \mathrm{O} & -2.44037800 & 0.91459300 & 2.87630100 \\ \mathrm{H} & -2.28314500 & 0.29656100 & 3.60811600 \\ \mathrm{O} & -0.44362300 & -1.10865700 & 0.58578800 \\ \mathrm{C} & -0.25351800 & -2.44377100 & 0.45179700 \\ \mathrm{O} & -1.08970200 & -3.25951000 & 0.82163200 \\ \mathrm{C} & 1.00398600 & -2.89955500 & -0.16743700 \\ \mathrm{C} & 2.22364900 & -2.36318600 & -0.43226700 \\ \mathrm{C} & 2.87819700 & -1.06288900 & -0.29957700 \\ \mathrm{C} & 2.23773600 & 0.15811300 & -0.02444300 \\ \mathrm{C} & 4.28064700 & -1.05151300 & -0.49244000 \\ \mathrm{C} & 2.96776300 & 1.34125200 & 0.06257600 \\ \mathrm{H} & 1.16845600 & 0.19927700 & 0.10604200 \\ \mathrm{C} & 5.01324100 & 0.12121900 & -0.39318100 \\ \mathrm{H} & 4.81276200 & -1.97175500 & -0.71647100 \\ \mathrm{C} & 4.35013600 & 1.33193700 & -0.11290300 \\ \mathrm{H} & 2.44933500 & 2.27599700 & 0.25986600 \\ \mathrm{O} & 6.36779700 & 0.10082500 & -0.57310700 \\ \mathrm{O} & 5.15759700 & 2.43256700 & -0.04453800 \\ \mathrm{H} & 4.63133300 & 3.22641500 & 0.14479000 \\ \mathrm{O} & -1.92277700 & 0.26530500 & -1.59310000 \\ \mathrm{H} & -2.29552500 & 0.52564300 & -2.45872400 \\ \mathrm{C} & -4.29887100 & 0.33952400 & -1.44451400 \\ \mathrm{O} & -4.32168700 & 0.42607900 & -2.65836900 \\ \mathrm{O} & -5.39203400 & 0.24336900 & -0.67957900 \\ \mathrm{H} & -6.17450400 & 0.26005800 & -1.26536900 \\ \mathrm{H} & -2.91305200 & -3.10986600 & -0.82708600 \\ \mathrm{H} & -2.95291600 & 2.56304100 & -0.64027100 \\ \mathrm{H} & -2.91473300 & -1.73042900 & -0.16991100 \\ \mathrm{H} & -1.81331400 & -1.42204700 & 2.08652400 \\ \mathrm{H} & -0.43225000 & 0.73105500 & 2.31120600 \\ \mathrm{H} & -1.61734300 & 2.77235400 & 1.48034200 \\ \mathrm{H} & & \\ \mathrm{H} & & & \end{array}$




\section{Scheme s2: Cartessian coordinates of the optimized parent compound of Luteolin-7-O-B- glucuronide}

51

\begin{tabular}{|c|c|c|c|}
\hline$C$ & -4.59144685 & -0.99689590 & -0.15878057 \\
\hline C & -3.20533845 & -0.82097667 & -0.04550461 \\
\hline $\mathrm{C}$ & -2.67728222 & 0.46770530 & 0.10285575 \\
\hline C & -3.52104289 & 1.57908372 & 0.19129311 \\
\hline $\mathrm{C}$ & -4.90187689 & 1.40640085 & 0.02611159 \\
\hline $\mathrm{C}$ & -5.44039772 & 0.11894531 & -0.12373790 \\
\hline H & -5.00091799 & -1.98018986 & -0.27010944 \\
\hline & -1.17479026 & 0.69116976 & 0.16292092 \\
\hline $\mathrm{H}$ & -5.54840379 & 2.25979994 & 0.02688197 \\
\hline C & -1.60551986 & 3.14238872 & 0.24402319 \\
\hline C & -0.69442448 & 2.13706607 & 0.18907477 \\
\hline $\mathrm{H}$ & 0.35255745 & 2.35921763 & 0.15820457 \\
\hline C & -1.12279976 & 4.59499034 & 0.07723673 \\
\hline & 0.24147258 & 4.86744926 & -0.09483360 \\
\hline & -2.04869649 & 5.64717562 & 0.10312762 \\
\hline C & 0.67987760 & 6.19218292 & -0.22268041 \\
\hline $\mathrm{H}$ & 0.94778910 & 4.06526307 & -0.12581273 \\
\hline C & -1.60970474 & 6.97096202 & -0.03165300 \\
\hline & -3.09066028 & 5.44002829 & 0.22620883 \\
\hline C & -0.24503445 & 7.24351283 & -0.19033543 \\
\hline & -2.31692837 & 7.77434690 & -0.01292760 \\
\hline & 0.20394092 & 8.59560209 & -0.31876947 \\
\hline $\mathrm{H}$ & 0.20981644 & 8.84305330 & -1.24601433 \\
\hline O & 2.07094293 & 6.47297996 & -0.38497542 \\
\hline $\mathrm{H}$ & 2.47341783 & 6.59540448 & 0.47788663 \\
\hline 0 & -3.01546722 & 2.90579407 & 0.47317461 \\
\hline 0 & -0.36518959 & -0.27218220 & 0.17232576 \\
\hline 0 & -2.32881723 & -1.95051545 & -0.08342069 \\
\hline $\mathrm{H}$ & -2.77696815 & -2.71811284 & 0.28180035 \\
\hline 0 & -6.85950120 & -0.04873986 & -0.22282761 \\
\hline C & -7.20163530 & -1.19479294 & -1.00857885 \\
\hline C & -6.77450513 & -0.99191081 & -2.50737021 \\
\hline C & -9.08343512 & -1.96750724 & -2.06051055 \\
\hline C & -7.79823786 & -0.06201702 & -3.07680372 \\
\hline C & -9.07897589 & -0.91662948 & -3.20459727 \\
\hline 0 & -8.64992654 & -1.34995280 & -0.89093736 \\
\hline 0 & -5.44469002 & -0.46890587 & -2.54787912 \\
\hline $\mathrm{H}$ & -5.18867882 & -0.31391154 & -3.45980876 \\
\hline 0 & -7.46604900 & 0.48345276 & -4.35479140 \\
\hline $\mathrm{H}$ & -8.17662280 & 1.05609002 & -4.65150628 \\
\hline 0 & -9.10835769 & -1.65993927 & -4.42666693 \\
\hline $\mathrm{H}$ & -9.91493357 & -2.17993172 & -4.46490584 \\
\hline C & -10.49856945 & -2.56144007 & -1.91510838 \\
\hline 0 & -10.90407584 & -2.94790621 & -0.78793063 \\
\hline 0 & -11.35273264 & -2.67705903 & -3.05567876 \\
\hline $\mathrm{H}$ & -11.93811447 & -3.42969256 & -2.93988210 \\
\hline $\mathrm{H}$ & -6.80408152 & -1.90131200 & -3.06853692 \\
\hline $\mathrm{H}$ & -7.91402084 & 0.73990628 & -2.37826846 \\
\hline $\mathrm{H}$ & -6.68680094 & -2.05462511 & -0.63512142 \\
\hline $\mathrm{H}$ & -8.38847279 & -2.75199618 & -2.27904030 \\
\hline $\mathrm{H}$ & -9.91589798 & -0.25090396 & -3.1734838 \\
\hline
\end{tabular}


